\title{
A New Semantics of Social Commitments using Branching Space-Time Logic
}

\author{
M. El-Menshawy \\ Depart. of Electrical and Computer Engineering \\ Concordia University, Montreal, Canada \\ m_elme@encs.concordia.ca
}

\author{
J. Bentahar, R. Dssouli \\ Concordia Institute for Inf. Sys. Engineering \\ Concordia University, Montreal, Canada \\ \{bentahar,dssouli\}@ciise.concordia.ca
}

\begin{abstract}
Commitments based on branching time logic are powerful representations for modeling multi-agent interactions. Current approaches into commitments have conceived these representations and evolved the commitments as "world-wide" states called moments. These approaches do not capture the space and space-like dimensions and ignore the causal relation between the participating agents. This paper presents a significant step towards developing a new logical semantics of social commitments based on Branching Space-Time (BST) logic. The contributions of this paper are threefold: first, we reformulate BST-logic from philosophical perspective to computational logic being used in computer science discipline; second, we enhance this logic with social commitments (propositional and conditional) and space-like modalities; and third, we present a new semantics model for social commitments and twoparty operations that manipulate commitments in the same framework.
\end{abstract}

Keywords-Branching Space-Time, Space-like Operator, Social Commitments.

\section{INTRODUCTION}

Recent years have seen an increasing interest in modeling agent interaction using computational logics [18], [19], [7], [11]. In this context, a formal semantics of social commitments based on logics for multiagent systems has been broadly acknowledged, specially for agent communication.

Some of interesting semantics frameworks such as (CTL, $C T L^{*}$ and $C T L^{ \pm}$) [12], [17], [6], [7], [20] have already been proposed. However, these frameworks don't capture the space and space-like dimensions in modeling and evolving the commitments and associated operations. Furthermore, the causal relation between agents exchanging commitments is not captured in these proposals. The causal relation means that when a social commitment is made by an agent towards another agent, the answer of the recipient will depend causally on the received commitment. Also, in CTLlike logics, the agent life cycle is not captured and its physical space is ignored. Consequently, the existence of communicating agents in the same (physical or virtual) space is not represented in these logics. Moreover, the operations that are used to manipulate social commitments are either disregarded, or captured as axioms (e.g., [12], [13], [10]) on the top of commitment semantics. However, the semantics of commitment operations themselves (e.g., create, fulfill) haven't been defined yet. This paper aims to reformulate another logic called Branching Space-Time (BST) introduced in [2], [3], [1] from philosophical perspective to computational logic in terms of accessibility relations within a Kripke structure to tackle the aforementioned shortcomings of branching time logics.

Our contributions in this paper are manifold: (1) we enhance BST-logic with three modalities for commitments, actions on the commitments and space-like operators; (2) a unified logical model capturing the semantic issues of social commitments and associated operations; (3) our framework captures the special and causal relationships between agents when they exchange and handle their commitments; and (4) our framework also defines agents' life cycles that limits agent actions in a relativistic space and time to occur and evolve locally. Thus the proposed semantics is fully defined in the same framework and not only captured by some enforced rules like in [12]. Additionally, our approach considers social sanctions as a methodology to incentive agents toward satisfying their commitments.

The remainder of this paper is organized as follows. Section II reviews rudiments of $B S T$ and agents life cycle. Section III presents the notion of social commitment and its formal notation. Section IV explains the syntax and semantics of the main elements in our logical model for commitments and associated operations. Section V compares our logical semantics with related work.

\section{OvERVIEW OF BRANCHING SPACE-TIME}

This section introduces some rudiments of $B S T$ from Belnap's works [1], [2], [3], [4] that satisfy the purpose of this paper. The $B S T$ theory combines nondeterminism (which is also the case of branching time logics) and relativistic space-time in a rigorous way. $B S T$ views spacetime as a set of point events subjected to a causal order. The structure of $B S T$ is defined essentially from two primitives: $\langle\mathbb{S}, \leq\rangle$ where:

- $\mathbb{S}$ is a nonempty set of states.

- $\leq$ is a partial causal ordering on $\mathbb{S}$.

In $B S T$, a history $h$ plays the same role like a path in branching time and is defined as a maximal and directed set of states. A set $\mathbb{E}$ is directed meaning that $\mathbb{E}$ contains an upper bound for each pair of its members. While maximal means that there is no $\mathbb{E}$ such that $h \subset \mathbb{E}[5]$. Thus each 
history satisfies the main features of Minkowski space-time. The set of all histories is denoted by $\sigma$ and the set of histories starting at a state $s_{i}$ is denoted by $\sigma^{s_{i}}$. Two histories $h_{1}$ and $h_{2}$ are undivided at a state $s_{i}$ and denoted by $h_{1} \approx_{s_{i}}$ $h_{2}$ iff $\exists s_{j} \in h_{1} \cap h_{2}: s_{i} \leq s_{j}$ and if $s_{i}$ is the maximal element in $h_{1} \cap h_{2}$ then $h_{1}$ divides from $h_{2}$ at $s_{i}$, which is called splitting point. The notion of path in branching time differs from the history in $B S T$. In branching time, a path corresponds to a maximal linear and infinite sequence of states through a treelike partial ordering of our world. In $B S T$, the history cannot be linearly ordered, because the states that are related by a space-like relation (explained in subsection $I V-B$ ) cannot be linearly ordered. Also, many states linearly ordered can belong to the same history. The intuition is that each line in this history corresponds to a life cycle part of a given agent in which each state corresponds to one and only one agent opposite to branching time where each state would be occupied by more than one agent. The set of agents is denoted by $\mathcal{A G \mathcal { T }}$ and the life cycle of an agent (reproduced from [4]) is defined as follows:

Definition 1: The life cycle of $A g \in \mathcal{A G \mathcal { T }}$ is a treelike set of states in which the root is the greatest lower bound for all states of $A g$ and is called the birth of $A g$. The intersection of history $h$ and $A g$ is the set of states linearly ordered where the least upper bound of this intersection is called the death of $A g$.

To express the fact that something happens in the same space-time state but in different histories, Muller has extended the framework of $B S T$ with an equivalence relation over states [14].

Definition 2: Let $h_{1}, h_{2} \in \sigma$ be two histories, $h_{1} \neq h_{2}$, and $s_{i}, s_{j}$ are two states in $\mathbb{S}$. Let $R_{h_{1}, h_{2}}$ be the region of overlap between $h_{1}$ and $h_{2}$. The equivalence relation $\approx \subseteq \mathbb{S} \times \mathbb{S}$ is defined by $s_{i} \in h_{1} \approx s_{j} \in h_{2}$ iff $s_{i}=s_{j}$ and $s_{i} \in R_{h_{1}, h_{2}}$. While, the equivalence class $\left[s_{i}\right]$ for state $s_{i}$ is defined by $\left[s_{i}\right]=\left\{s_{j}: s_{i} \approx s_{j}, s_{i} \in h_{1}, s_{j} \in h_{2}\right.$ and $\left.h_{1} \neq h_{2}\right\}$.

The $R_{h_{1}, h_{2}}$ is a set of states in the causal past of splitting state between two histories. We can easily prove that the relation $\approx$ is reflexive, symmetric and transitive. We can also prove that $\approx$ satisfies two conditions: $(1)$ the intersection between a history and an equivalence class contains exactly one element and $(2)$ the equivalence relation $\approx$ respects the ordering $(=,<,>)$. Below, we explain the notion of commitments.

\section{Social Commitments}

A commitment is an engagement made by one agent, the debtor, and directed towards another agent, the creditor, in the same space so that some fact is true. The commitments are contextual, manipulable and possibly conditional [16], then they are unlike obligations. Furthermore, commitments are social and observable by all the participants. Consequently, social commitments $(S C)$ are different from the agent's private mental states like beliefs, desires and intentions. In real-life settings a violation can occur if agents are malicious or for any other reason. Bellow two types of social commitments:

Definition 3: Propositional social commitment is a predicate $S C^{p}\left(i d, A g_{1}, A g_{2}, \phi\right)$ where $i d$ is the commitment's identifier, $A g_{1}$ is the debtor, $A g_{2}$ is the creditor, and $\phi$ is the commitment's content.

Definition 4: Conditional social commitment is a predicate $S C^{c}\left(i d, A g_{1}, A g_{2}, \tau, \phi\right)$ where $i d, A g_{1}, A g_{2}, \phi$ have the same meaning as in Definition 3and $\tau$ is the condition.

The conditional commitment is transformed to the propositional commitment when the condition $\tau$ is true. Our model assumes that every commitment must have a unique identifier to help in distinguishing it from others.

\section{A. Social commitment life cycle}

In this section we present the life cycle of commitment to distinguish the relationships between commitments' statuses. The commitment could be conditional or unconditional (i.e., propositional). This is represented by the selection operator. The first operation an agent can perform on a commitment is Creation. When created, a conditional commitment can move to the state of created unconditional commitment if the condition is true otherwise the conditional commitment moves to the final state. When the unconditional commitment is created, then it may move to one of the following states: fulfilled, violated, or withdrawn. The commitment can be withdrawn if the debtor decides to cancel it without any sanction from the creditor. The commitment is fulfilled if commitment's content is satisfied by the debtor and it will receive the reward from the creditor. The social commitment is violated if commitment's content is violated by the debtor and it will pay the penalty to the creditor.

\section{Logical Model of Social Commitments}

This section introduces the syntax and semantics of different elements in our formal language $\mathcal{L}$. This propositional language enhances $B S T$ [14], [1], [2] with modalities $S C^{p}$ for propositional, $S C^{c}$ for conditional commitments, $S P$ for space-like operator, and Act for actions applied to commitment. The resulting logic is called $B S T^{s c}$. The time is discrete and branching in future to represent all choices available to participating agents in conversations and linear in the past. Moreover, the dynamic behavior of agents are captured by actions that manipulate commitments.

\section{A. Syntax}

Let $\Phi_{p}$ be a set of atomic propositions and $\mathcal{A G \mathcal { T }}$ a set of agent names. $\mathcal{A C T}$ is a set of actions used to manipulate commitment. $L$ and Act are nonterminals corresponding to $\mathcal{L}$ and $\mathcal{A C T}$, respectively. We also use the following 
conventions: $i d_{0}, i d_{1}$, etc. are unique commitment identifiers, $A g_{1}, A g_{2}$, etc. are agent names, $p, q$, etc. are atomic propositions in $\Phi_{p}$ and $\phi, \psi$, etc. are formulae in $\mathcal{L}$. Table I gives the formal syntax of $\mathcal{L}$ expressed in Backus-Naur Form (BNF) grammar where" $\rightarrow$ " and "|" are meta-symbols.

$$
\begin{aligned}
& \text { L1. } L \rightarrow C \mid \text { Act }|S P L| p|\neg L| L \vee L\left|X_{c}^{+} L\right| X_{c}^{-} L \\
& \left|L U_{c}^{+} L\right| L U_{c}^{-} L|A L| E L \\
& \text { L2. } C \rightarrow S C^{p}(i d, A g, A g, L) \mid S C^{c}(i d, A g, A g, L, L) \\
& \text { L3. Act } \rightarrow \text { Create }(A g, C)|W i t h d r a w(A g, C)| \operatorname{Fulfill}(A g, C) \\
& \text { | Violate }(A g, C) \\
& \text { Table I }
\end{aligned}
$$

The intuitive meanings of the causal operators are straightforward from CTL* with respect to the structure of EinsteinMinkowski space-time, where causal next $\left(X_{c}^{+}\right)$, causal previous $\left(X_{c}^{-}\right)$, causal until $\left(U_{c}^{+}\right)$, and causal since $\left(U_{c}^{-}\right)$ are the names of these operators respectively. Informally, $A$ and $E$ are the universal and existential history-quantifiers over the set of all histories starting from the current moment. Furthermore, there are some useful abbreviations based on temporal operators $\left(X_{c}^{+}, X_{c}^{-}, U_{c}^{+}, U_{c}^{-}\right)$: (sometimes in the causal future) $F_{c}^{+} \phi \triangleq$ true $U_{c}^{+} \phi$; (sometimes in causal past) $F_{c}^{-} \phi \triangleq t r u e U_{c}^{-} \phi$; (globally in causal future) $G_{c}^{+} \phi \triangleq$ $\neg F_{c}^{+} \neg \phi$ and (globally in causal past) $G_{c}^{-} \phi \triangleq \neg F_{c}^{-} \neg \phi$.

\section{B. Semantics}

We define the $B S T$ frame as a structure $\mathbb{F}=\left\langle\mathbb{S}, R_{M}, \approx\right\rangle$ where $\mathbb{S}$ is a set of possible states of our world; $R_{M} \subseteq$ $(\mathbb{S} \times \mathbb{S})$ is a total transition relation; and $\approx \subseteq(\mathbb{S} \times \mathbb{S})$ is the equivalence relation on $\mathbb{S}$. To model $B S T$ each state is a vector in $\mathbf{R}^{2}$ where the first dimension represent space and the second refers to time. Let $s_{i}=\left(x_{1}, y_{1}\right)$ and $s_{j}=$ $\left(x_{2}, y_{2}\right)$ be space-time states then the space-time distance between $s_{i}$ and $s_{j}$ is defined by $D_{M}^{2}\left(s_{i}, s_{j}\right)=-\left(x_{1}-\right.$ $\left.x_{2}\right)^{2}+\left(y_{1}-y_{2}\right)^{2}$. According to this distance, BST defines three relations between any two states: time-like relation, $s_{i} \leq_{M} s_{j}$ iff $D_{M}^{2}\left(s_{i}, s_{j}\right)<0 \& x_{1} \leq x_{2}$ (where " $\leq_{M}$ " denotes Einstein-Minkowski causal order on $\mathbf{R}^{2}$ ); space-like relation, $s_{i} \Varangle_{M} s_{j} \& s_{j} \Varangle_{M} s_{i}$ iff $D_{M}^{2}\left(s_{i}, s_{j}\right)>0$; and light-like relation, $D_{M}^{2}\left(s_{i}, s_{j}\right)=0$. Furthermore, if there exists a transition $\left(s_{i}, s_{j}\right) \in R_{M}$, then we have $s_{i} \leq_{M} s_{j}$ but the reverse isn't always true.

Our formal model for language $\mathcal{L}$ is based on a Kripkelike structure with five-tuple $M=\left\langle\mathbb{F}, \mathbb{V}, \mathbb{F}_{\text {sag }}, \mathbb{R}_{s c p}, \mathbb{R}_{s c c}\right\rangle$ where:

- $\mathbb{F}$ is a $B S T$ frame.

- $\mathbb{V}: \Phi_{p} \rightarrow 2^{(\mathbb{S} \times \sigma)}$ is a valuation function assigning to each atomic proposition a set of states in histories when the proposition is true.

- $\mathbb{F}_{\text {sag }}: \mathbb{S} \rightarrow \mathcal{A G} \mathcal{T}$ is a function used to return the agent currently located or perviously located at a given state.

- $\mathbb{R}_{s c p}: \mathbb{S} \times \mathbb{S} \times \sigma \rightarrow 2^{\sigma}$ is a function producing an accessibility modal relation for propositional commitments.
- $\mathbb{R}_{s c c}: \mathbb{S} \times \mathbb{S} \times \sigma \rightarrow 2^{\sigma}$ is a function producing an accessibility modal relation for conditional commitments. The function $R_{s c p}$ associates to each two states $s_{i}$ and $s_{j}$ along the history $h$ the set of histories along which an agent commits towards another agent. These histories are conceived as merely "possible" where the commitments' contents made at state $s_{i}$ in the causal past of state $s_{j}$ are true. For example, if we have: $h^{\prime} \in R_{s c p}\left(s_{i}, s_{j}, h\right)$ this means that the commitments made at state $s_{i}$ by a given agent towards another given agent at state $s_{j}$ in its causal past is satisfied within the accessible history $h^{\prime} . R_{s c c}$ is similar to $R_{s c p}$ and it gives us the histories along which the resulting unconditional commitment is satisfied if the underlying condition is true. If $h$ is a history starting at a given state $s_{i}$, then the prefix of $h$ starting at a state $s_{j}$ $\left(s_{j} \leq_{M} s_{i}\right)$ is a history denoted by $h \downarrow s_{j}$ and suffix of $h$ starting at state $s_{k}\left(s_{i} \leq_{M} s_{k}\right)$ is a history denoted by $h \uparrow s_{k}$. The state $s_{k}$ is in the causal future of $s_{i}$ over the history $h$ such that $h$ is part of $h \uparrow s_{k}$. If $s_{i}$ is a state, then we assume that $s_{i-1}$ is the previous state in the linear causal past $\left(\left(s_{i-1}, s_{i}\right) \in R_{M}\right)$ and $s_{i+1}$ is the causal next state on a given history $\left(\left(s_{i}, s_{i+1}\right) \in R_{M}\right)$.

To define the semantics, we use the following metasymbols: \& means "and", $\Rightarrow$ means "implies that" and the expression $M,\left\langle s_{i}, h\right\rangle \models \phi$ means that "the model $M$ satisfies $\phi$ along accessible history $h$ starting at $s_{i}$ where there is only one agent located at $s_{i}$ ". Furthermore, $s_{i} \leq_{M} s_{j}$ captures the causal relation between the addressee and sender [3] to exchange commitments. Table II illustrates our formal semantics.

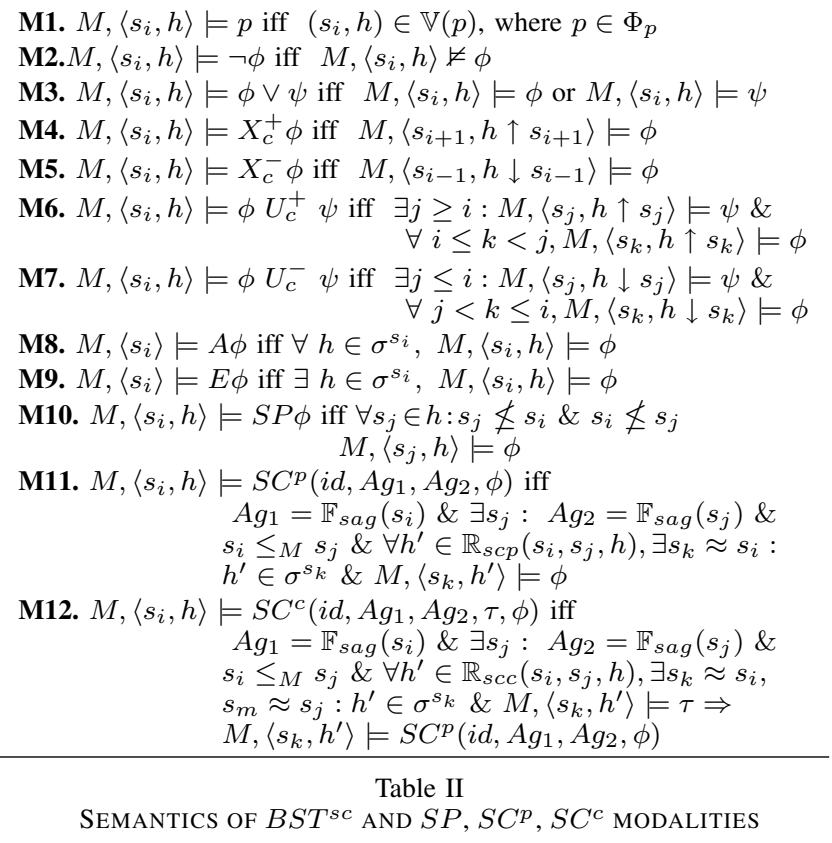

The semantics of the operators from $M 1$ to $M 9$ is straight forward from $\mathrm{CTL}^{*}$ with respect to the structure 
of Minkowski space-time. $M 10$ presents the semantics of space-like operator in the model $M$ at state $s_{i}$ along history $h$ iff the content $\phi$ is true in all states in the same history without having causal time-like with $s_{i}$. M11 gives the semantic of propositional commitment, where it is satisfied in the model $M$ at state $s_{i}$ along history $h$ iff the content $\phi$ is true in all accessible histories $h^{\prime}$ starting at an equivalent state $s_{k}$ to $s_{i}$. In fact, $h$ and $h^{\prime}$ start at two equivalent states, which means that they start at the same moment. Similarly, M12 gives the semantics of conditional commitment, where it is satisfied in the model $M$ at state $s_{i}$ along history $h$ iff in all accessible histories $h^{\prime}$, if the condition $\tau$ is true, then the underlying unconditional commitment is also true.

1) Semantics of actions on social commitments:

We define the semantics of two-party operations (i.e., create, withdraw, fulfill, and violate) that depend only on two agents. The context of these operations are given in [16], [12], [13]. To simplify the notations used in the semantics, we suppose that the actions are momentary and their formulae are evaluated over histories.

Creation action: the semantics of creation action of a propositional commitment (see Table III) is satisfied in the model $M$ at state $s_{i}$ along history $h$ iff the debtor performs the creation action resulting in establishing commitment along history $h$ (i.e., creating and revealing commitment holds in the same state); and (ii)the created commitment was not established in the causal past at the state previously occupied by agent $A g_{1}$.

$$
\begin{aligned}
& \text { M13. } M,\left\langle s_{i}, h\right\rangle \models \text { Create }\left(A g_{1}, S C^{p}\left(i d, A g_{1}, A g_{2}, \phi\right)\right) \text { iff } \\
& \begin{array}{c}
\text { (i) } A g_{1}=\mathbb{F}_{\text {sag }}\left(s_{i}\right) \& M,\left\langle s_{i}, h\right\rangle \models S C^{p}\left(i d, A g_{1}, A g_{2}, \phi\right) \& \\
(i i) \forall s_{0} \in \mathbb{S}: s_{0} \leq M s_{i} \& A g_{1}=\mathbb{F}_{\text {sag }}\left(s_{0}\right) \\
\Rightarrow M,\left\langle s_{0}, h\right\rangle \models \neg S C^{p}\left(i d, A g_{1}, A g_{2}, \phi\right)
\end{array} \\
& \text { Table III } \\
& \text { SEMANTICS OF CREATION ACTION OF } S C^{p}
\end{aligned}
$$

The semantics of creation action of a conditional commitment (see Table IV) is defined in the same way.

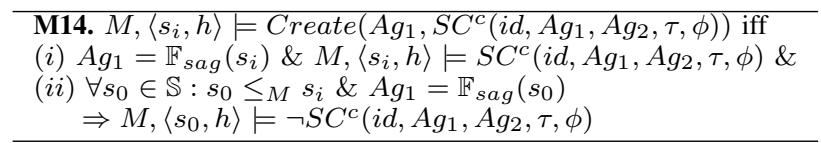

\section{Table IV}

SEMANTICS OF CREATION ACTION OF $S C^{c}$

Withdrawal action: the semantics of withdrawal action of a propositional commitment (see Table V) is satisfied in the model $M$ at state $s_{i}$ along history $h$ iff (i) the commitment was created in the past at $s_{0}$ through the prefix $h \downarrow s_{0}$; and (ii) this prefix is not one of the accessible histories using $\mathbb{R}_{s c p}$ and at the same time there is still a possibility of satisfying the commitment since there is a history $h^{\prime}$ whose the prefix is still accessible using $\mathbb{R}_{s c p}$ (see Figure 1).

Fulfillment action: the semantics of fulfillment action (see Table VI) is defined in the same line as withdrawal. In (i), the prefix $h \downarrow s_{0}$ of the current history $h$ (along which the commitment has been created) is accessible using $\mathbb{R}_{\text {scp }}$; and (ii) at the current state $s_{i}$ there is still a possible choice of not satisfying the commitment since a non-accessible history exists. We notice that being accessible means that the content $\phi$ is true along $h \downarrow s_{0}$. As for withdrawal, fulfillment action makes sense only when a non-fulfilment action is still possible.
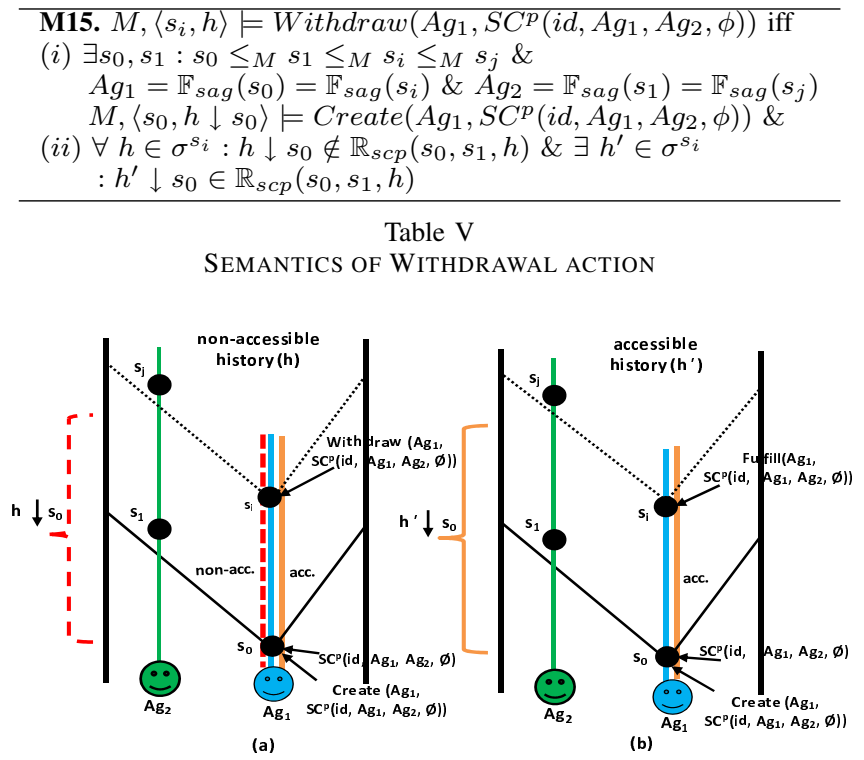

Figure 1. (a) withdrawal action and (b) the possibility to fulfill commitment.

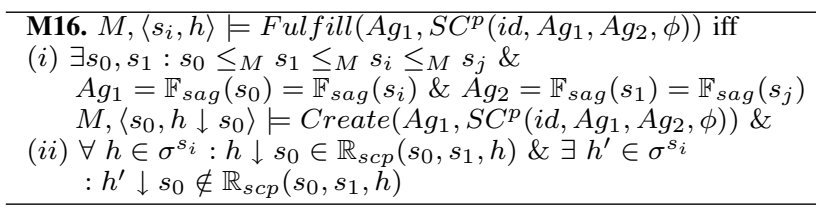

Table VI

SEMANTICS OF FULFILLMENT ACTION

Violation action: the semantics of violation action (see Table VII) is almost similar to the semantics of withdrawal action. The main difference is related to the fact that the commitment content is false in case of violation (i).

$$
\begin{aligned}
& \text { M17. } M,\left\langle s_{i}, h\right\rangle \models \operatorname{Violate}\left(A g_{1}, S C^{p}\left(i d, A g_{1}, A g_{2}, \phi\right)\right) \text { iff } \\
& (i) \exists s_{0}, s_{1}: s_{0} \leq_{M} s_{1} \leq_{M} s_{i} s_{j} \& \\
& A g_{1}=\mathbb{F}_{\text {sag }}\left(s_{0}\right)=\mathbb{F}_{\text {sag }}\left(s_{i}\right) \& A g_{2}=\mathbb{F}_{\text {sag }}\left(s_{1}\right)=\mathbb{F}_{\text {sag }}\left(s_{j}\right) \\
& M,\left\langle s_{0}, h \downarrow s_{0}\right\rangle \models C r e a t e\left(A g_{1}, S C^{p}\left(i d, A g_{1}, A g_{2}, \phi\right)\right) \& \\
& (i i) \forall h \in \sigma^{s_{i}}: M,\left\langle s_{0}, h \downarrow s_{0}\right\rangle \models \neg \phi \& \\
& \exists h^{\prime} \in \sigma^{s_{i}}: h^{\prime} \downarrow s_{0} \in \mathbb{R}_{s c p}\left(s_{0}, s_{1}, h\right) \\
& \text { Table VII } \\
& \text { SEMANTICS OF VIOLATION ACTION }
\end{aligned}
$$

The fact that $\phi$ is false implies that $h \downarrow s_{0}$ is not accessible, but the reverse is not always true as explained above. We notice that violation makes sense when a choice of satisfying the commitment is still possible at the current state (ii). 


\section{Discussion And Related Work}

Social commitments and their semantics have been widely investigated during the last years [6], [7], [16], [17], [12], [20], [9], [15], whilst the semantics developed in this paper is different from all of them and directed to tackle some ignored issues in the previous framework. Following is the comparison of our approach with related works.

The proposed semantics of conditional commitment is different from the [15]'s in which conditional commitments are considered as intentions, while our commitments are social and different from private intentions. Also, in our semantics the conditional commitments are transformed to propositional commitments in all accessible histories when the underlying condition is true, which is not captured in [15]. The semantics we give to the associated operations is also different from the semantics proposed in [10] using $L T L$ to define these operations based on some postulates to overcome the issues of autonomy in distributed systems. Furthermore, the semantics of propositional commitment differs from the one proposed in [8] where the propositional commitment is fulfilled when the creditor does not believe that the commitment content is false and cannot challenge it anymore. Also the authors define the satisfaction of this semantics by considering beliefs as public states. The violation operation is completely disregarded in [12], [13], [10].

Our semantics of commitment operations doesn't suffer from the "recursion problem" revealed in [7], which leads to "state explosion" problem in the earlier phases of model checking. This work is based on Belnap's BST and agency theories [3], [4]. In addition, this paper enhances $B S T$ in the sense that it defines the formal semantics of the CTL-like temporal operators "next and until" in $B S T$ and investigates its application for multiagent systems and agent communication. Furthermore, Belnap has proposed norms combined with strategies to capture the notion of obligations between agents in the form of "a declarative core in direct speech". However, the notion of social commitments developed here is more flexible than direct obligations/prohibitions and more rigid than permissions.

This work opens many future opportunities since it's the first essay on using BST for multiagent systems. We are planning to study other types of social commitments as proposed in [7] and investigate more features of $B S T$ theory like causal probability. We also plan to introduce a useful theory of games in $B S T$ with the causal structure between players.

\section{ACKNOWLEDGEMENTS}

We would like to thank NSERC (Canada), NATEQ and FQRSC (Québec) for their financial support.

\section{REFERENCES}

[1] N. Belnap. Branching space-time. Synthese, vol.92, pp. 385434, 1992.
[2] N. Belnap. A theory of causation: Causae causantes (originating causes) as inus conditions in branching space-times. British Journal for the Philosophy of Science, vol. 56, pp. 221-253, 2005.

[3] N. Belnap. Agents and agency in branching space-times. Logic, Thought and Action, Springer, Vol. 2, IV, pp. 291-313, 2006.

[4] N. Belnap. Prolegomenon to norms in branching space-times. The Int. Conf. on Deontic Logic in Comp. Science (DEON'08), Luxembourg, 2008.

[5] N. Belnap. From newtonian determinism to branching-spacetime indeterminism, Logic, Concepts, and Principles of Action. (eds.) Thomas Muller and Albert Newen, Verlag GmbII, pp. 13-31, 2007.

[6] J. Bentahar, B. Moulin, J-J Meyer and B. Chaib-draa. A logical model for commitment and argument network for agent communication (Extended Abstract). In Proc. of 3rd Int. Joint Conf. on AAMAS, pp. 792-799, 2004. ACM Press.

[7] J. Bentahar, B. Moulin, J-J.Ch. Meyer and Y. Lespérance. A new logical semantics for agent communication. K. Inoue, $\mathrm{K}$. Satoh, F. Toni (eds.), Comput. Logic in Multi-Agent Sys., LNAI 4371, pp. 151-170, 2007. Springer.

[8] G. Boella, R. Damiano, J. Hulstijn and L. Torre. Distinguishing propositional and action commitment in agent communication, In Proc. of the Workshop on Comput. Models of Natural Argument (CMNA'07).

[9] A.K. Chopra, and M.P. Singh. Constitutive interoperability. In Proc. of the Int. Joint Conf. on AAMAS, pp. 797-804, 2008.

[10] A.K. Chopra, and M.P. Singh. Multiagent commitment alignment. In Proc. of the 8th Int. Joint Conf. on Autonomous Agents and MultiAgent Sys. (AAMAS). May 2009.

[11] U. Endriss, N. Maudet, F. Sadri and F. Toni. Protocol conformance for logic-based agents. In Proc. of the 18th Int. Joint Conf. on AI (IJCAI), pp. 679-684, 2003.

[12] A.U. Mallya, P. Yolum, and M.P. Singh. Resolving commitments among autonomous agents. F. Dignum (eds.), Advances in Agent Commu., LNAI vol.2922, pp. 166-182, 2004.

[13] A.U. Mallya, and M.P. Singh. An algebra for commitment protocols. Journal of Autonomous Agents and Multi-Agent Sys., vol. 14, pp. 143-163, 2007.

[14] T. Muller. Branching space-time, modal logic and the counterfactual conditional. T. Placek and J.Butterfield (eds.), Nonlocality and Modality, Klumer, pp.273-291, 2002.

[15] M.K. Shakil and L. Yves. On the semantics of conditional commitment. In Proc. of the 5th Int. joint Conf. on Auto. agents and multiagent sys., AAMAS, pp.1337-1344, 2006.

[16] M.P. Singh. An ontology for commitments in multiagent systems: toward a unification of normative concepts. AI and Law, vol.7, pp.97113, 1999.

[17] M.P. Singh. A social semantics for agent communication language, Issues in Agent Communication. F. Dignum and M. Greaves (eds.), LNAI 1916, pp.31-45, 2000.

[18] F. Toni. Multi-agent systems in computational logic: challenges and outcomes of the SOCS project. F. Toni, P. Torroni (eds.), Comput. Logic in Multi-Agent Sys. Springer LNAI 3900, pp. 420-426, 2006.

[19] F. Toni and J. Bentahar. Computational logic-based agents. In Autonomous Agents and Multi-Agent Systems, Vol.16, No.3, pp. 211-213, 2008, Springer.

[20] M. Verdicchio and M. Colombetti. A logical model of social commitment for agent communication. In Proc. of the Int. Joint Conf. on AAMAS, pp. 528-535, 2003. 